\title{
Left Dorsal Premotor Cortex and Supramarginal Gyrus Complement Each Other during Rapid Action Reprogramming
}

\author{
Gesa Hartwigsen, ${ }^{1,2}$ Sven Bestmann, ${ }^{3}$ Nick S. Ward,${ }^{3,4}$ Saskia Woerbel, ${ }^{1}$ Claudia Mastroeni, ${ }^{1}$ Oliver Granert, ${ }^{1}$ \\ and Hartwig R. Siebner ${ }^{1,5}$ \\ ${ }^{1}$ Department of Neurology, Christian-Albrechts University Kiel, 24105 Kiel Germany; ${ }^{2}$ Language and Aphasia Laboratory, Department of Neurology, \\ University Medical Centre of Leipzig, 04103 Leipzig, Germany; ${ }^{3}$ Sobell Department of Motor Neuroscience and Movement Disorders, UCL Institute of \\ Neurology, University College of London, WC1N 3BG London, United Kingdom; ${ }^{4}$ Department of Brain Repair and Rehabilitation, UCL Institute of \\ Neurology, University College London, WC1N 3BG London, United Kingdom; and ${ }^{5}$ Danish Research Centre for Magnetic Resonance, Copenhagen \\ University Hospital Hvidovre, DK-2650, Hvidovre, Denmark
}

The ability to discard a prepared action plan in favor of an alternative action is critical when facing sudden environmental changes. We tested whether the functional contribution of left supramarginal gyrus (SMG) during action reprogramming depends on the functional integrity of left dorsal premotor cortex (PMd). Adopting a dual-site repetitive transcranial magnetic stimulation (rTMS) strategy, we first transiently disrupted PMd with "off-line" 1 Hz rTMS and then applied focal "on-line" rTMS to SMG while human subjects performed a spatially precued reaction time (RT) task. Effective on-line rTMS of SMG but not sham rTMS of SMG increased errors when subjects had to reprogram their action in response to an invalid precue regardless of the type of preceding off-line rTMS. This suggests that left SMG primarily contributes to the on-line updating of actions by suppressing invalidly prepared responses. On-line rTMS of SMG additionally increased RTs for correct responses in invalidly precued trials, but only after off-line rTMS of PMd. We infer that off-line rTMS caused an additional dysfunction of PMd, which increased the functional relevance of SMG for rapid activation of the correct response, and sensitized SMG to the disruptive effects of on-line rTMS. These results not only provide causal evidence that left PMd and SMG jointly contribute to action reprogramming, but also that the respective functional weight of these areas can be rapidly redistributed. This mechanism might constitute a generic feature of functional networks that allows for rapid functional compensation in response to focal dysfunctions.

\section{Introduction}

In an ever-changing world, the ability to rapidly adjust ones action to sudden changes in the environment is important for survival. Reacting to changes requires the cancellation of the present action plan and its replacement by a new appropriate action. Previous work has identified several frontal and parietal cortical areas that contribute to the efficient reprogramming of actions (Chambers et al., 2007; Forstmann et al., 2008b; Mars et al., 2009; Neubert et al., 2010; Verbruggen et al., 2010). For instance, it has been shown that the left dorsal premotor cortex (PMd) is prefer-

Received Feb. 29, 2012; revised Aug. 15, 2012; accepted Sept. 13, 2012.

Author contributions: G.H., S.B., N.S.W., and H.R.S. designed research; G.H., S.W., C.M., and 0.G. performed research; G.H. analyzed data; G.H., S.B., N.S.W., and H.R.S. wrote the paper.

This work was supported by the Volkswagenstiftung Grant no. I/79-932 (H.R.S.). G.H. is supported by the Deutsche Forschungsgemeinschaft (HA 6314/1-1). S.B. is supported by the Biotechnology and Biological Sciences Research Council and European Research Council (ActSelectContext 260424). H.R.S. was supported by the Bundesministerium für Bildung und Forschung (01G00511 "NeurolmageNord") and a Grant of Excellence sponsored by The Lundbeck Foundation Mapping, Modulation and Modeling the Control of Actions (ContAct) (R59 A5399).

Correspondence should be addressed to Professor Hartwig R. Siebner, Danish Research Centre for Magnetic Resonance, Center for Functional and Diagnostic Imaging and Research Copenhagen University Hospital Hvidovre, Kettegaard Allé 30 DK-2650, Hvidovre, Denmark. E-mail: hartwig.siebner@drcmr.dk.

DOI:10.1523/JNEUROSCI.1010-12.2012

Copyright $\odot 2012$ the authors $\quad 0270-6474 / 12 / 3216162-10 \$ 15.00 / 0$ entially involved in nonroutine stimulus-response mapping (Iacoboni et al., 1998; Dassonville et al., 2001) or more indirect stimulus-response mapping based on arbitrary relations between cue and action (Hoshi and Tanji, 2006).

One way of addressing the role of a brain region for action reprogramming is repetitive transcranial magnetic stimulation (rTMS). rTMS provides an effective means to condition (off-line rTMS approach) or to perturb (on-line rTMS approach) distinct cortical regions (Siebner and Rothwell, 2003; Siebner et al., 2009). Both on-line and off-line rTMS have been successfully used to study the functional contribution of premotor or parietal areas to action selection and reprogramming (Rushworth et al., 2001; Chambers et al., 2007; O’Shea et al., 2007; Buch et al., 2010). These studies have provided some evidence that left supramarginal gyrus (SMG) and PMd contribute to motor reprogramming in response to spatial cues. On-line perturbation of left SMG with high-frequency rTMS prolonged action reprogramming in a spatially precued reaction time (RT) task (Rushworth et al., 2001). Low-frequency off-line rTMS of left PMd improved action reprogramming in a spatially precued RT task, reducing the number of errors in invalidly precued trials (Ward et al., 2010). In that study, functional magnetic resonance imaging during task per- 
formance revealed increased functional coupling between left PMd and SMG after $1 \mathrm{~Hz}$ rTMS over PMd, which correlated with the individual decrease in error rate (ER; Ward et al., 2010). However, the causal relevance of an intact interaction between PMd and SMG during action reprogramming remains unclear. Motivated by these previous studies, we used a dual-site offline- on-line rTMS approach to investigate how the functional contribution of left SMG to action reprogramming changes in the presence of a dysfunctional left PMd.

We reasoned that both PMd and SMG are key regions for nonroutine responses that require the integration of conflicting information during action reprogramming. Based on previous studies, we hypothesized that left SMG would be more critical for switching motor plans (Rushworth et al., 2001), by suppressing the release of a prepared but incorrect response triggered by an invalid precue. In contrast, PMd might be more critically involved in rapid stimulus response mapping requiring the initiation of the correct response (Christensen et al., 2007; Schubotz, 2007). If this were the case, online rTMS of left SMG should primarily interfere with the suppression of the prepared but incorrect response, and thus affect accuracy on invalidly precued trials. Moreover, interference with left PMd function should limit the brain's ability to compensate for the disruptive effect of SMG rTMS on stimulus-driven response activation. Therefore, after off-line rTMS of left PMd, subsequent on-line rTMS of left SMG should additionally delay the speed of responding to the unprepared response cue.

\section{Materials and Methods}

Subjects. 20 volunteers ( 9 females, mean age $=25.2$ years, age range $=21-30$ years) with no history of neurological disorders or head injury participated in the main experiment comparing the effects of dual-site TMS of both PMd and SMG. For comparing the effects of dual-site TMS of ventral premotor cortex (PMv) and SMG, a subset of 12 subjects was reexamined after 3-4 months with identical experimental procedures. A different subset of six subjects was reexamined to test the effects of focal on-line rTMS over PMv after 6 months to minimize repetition and familiarity effects. All subjects were right-handed (laterality index of $>80 \%$ ) according to the Edinburgh Handedness Inventory (Oldfield, 1971) and naive to TMS. During the experiments, subjects were equipped with earplugs. Written informed consent was obtained before the experiment. The study was performed according to the guidelines of the Declaration of Helsinki and approved by the Ethics Committee of the Medical Faculty of the Christian-Albrechts University of Kiel.

Experimental design and task. Figure 1 illustrates the study design. After a short training session, we reversibly perturbed activity in left PMd or vertex through low-frequency $1 \mathrm{~Hz}$ rTMS before the task (off-line rTMS; Figure $1 A, C$ ) in two separate sessions. Control condition was $1 \mathrm{~Hz}$ rTMS over the vertex. After $1 \mathrm{~Hz}$ off-line rTMS, participants performed a spatially precued RT task. The task required spatially congruent button presses, as quickly and accurately as possible, in response to a visually presented right- or left-sided target stimulus (S2). A directional precue (S1) indicated the probable position of the target stimulus. In $75 \%$ of the trials, $\mathrm{S} 1$ validly predicted the position of $\mathrm{S} 2$ and subjects were instructed to prepare for the response indicated by $\mathrm{S} 1$ (Fig. $1 B$ ). The task consisted of $2 \times 240$ trials: $2 \times 180$ trials with right valid or left valid precues and $2 \times 60$ trials with right invalid and left invalid precues (Fig. $1 B$ ). The intertrial interval was $1 \mathrm{~s}$.

During the task, four-pulse bursts of focal high-frequency $10 \mathrm{~Hz}$ rTMS were applied to left SMG on $50 \%$ of trials (vs $50 \%$ sham TMS trials), starting $20 \mathrm{~ms}$ after the presentation of the cue (on-line rTMS, Fig. 1C,D). Stimulus presentation and response recording was obtained using E-PRIME software (Psychology Software Tools).

TMS. Biphasic TMS pulses were applied using two P-Stim 160 stimulators connected to a figure-of-eight shaped coil (type Q.C.) with each half-wing having a diameter of $90 \mathrm{~mm}$ (Mag \& More GmbH). The TMS protocols were within the published safety limits (Rossi et al., 2009).
Stimulation intensity was set to $90 \%$ of individual resting motor threshold of the right first dorsal interosseous muscle for off-line and on-line rTMS. Neuronavigation was used to position the TMS coils and maintain their exact location and orientation throughout the experiment (see below). Each participant received $30 \mathrm{~min}$ of $1 \mathrm{~Hz}$ off-line rTMS (1800 stimuli) either over left PMd or vertex. We chose the vertex as control site because this area is associated with leg movements rather than hand movements and has been previously introduced as valid control condition in different motor studies (Bestmann et al., 2002; Grefkes et al., 2010; Ward et al., 2010). The two off-line rTMS sessions were applied in counterbalanced order at least $5 \mathrm{~d}$ apart. The off-line rTMS protocol was identical to the protocol used by Siebner et al. (2003) because it resulted in a suppression of neuronal activity in the stimulated PMd and connected brain regions for at least $1 \mathrm{~h}$ after the end of stimulation (Siebner et al., 2003; O'Shea et al., 2007). Off-line rTMS targeted left PMd using the mean stereotactic coordinates from a recent rTMS-functional magnetic resonance imaging study (Ward et al., 2010) (Fig. 1C).

Focal on-line rTMS consisted of a four-pulse train of effective or sham $10 \mathrm{~Hz}$ rTMS. The $10 \mathrm{~Hz}$ rTMS train was applied over left SMG $20 \mathrm{~ms}$ after the onset of S2 (Fig. 1D). Since the on-line rTMS protocol was motivated by a previous study targeting left SMG with high-frequency rTMS (Rushworth et al., 2001), we used the mean stereotactic coordinates reported in that study (Fig. 1C). Stereotactic coordinates for left PMv $(x, y, z=-62,4$, $20 \mathrm{~mm}$; MNI space) were obtained from a recent meta analysis (Mayka et al., 2006). Effective and sham rTMS trials were equally frequent (50\% each) and pseudorandomly intermingled. Sham on-line rTMS was given through an additional coil, which was placed over the first coil at an angle of $90^{\circ}$. Stimulation intensity was set $15 \%$ higher for sham as compared with effective on-line rTMS to produce a comparable acoustic stimulus without effectively stimulating the brain.

Neuronavigation. Neuronavigated TMS (TMS-Navigator; Localite) was based on the registered individual T1-weighted MR image. Before the experiment, a high-resolution MRI scan of the whole brain was acquired for each subject using an MPRAGE sequence in sagittal orientation (slice thickness 1 $\mathrm{mm}$; in-plane resolution $1 \times 1 \mathrm{~mm}$; TE/TR $=3.78 / 8.25 \mathrm{~ms}$ ). Individual stimulation sites were determined by calculating the inverse of the normalization transformation and transforming the mean coordinates derived from the studies described above from standard to "individual" space for each subject. To this end, individual T1-weighted images were segmented using the standard tissue probability maps provided in the Statistical Parametric Mapping software (SPM 5; http://www.fil.ion.ucl.ac.uk/spm/) implemented in MATLAB 7.7 (Mathworks) (Friston et al., 1995) with a very light bias regularization. Afterward, the resulting image was normalized and resampled to $1 \times 1 \times 1 \mathrm{~mm}^{3}$ voxels. A recently developed algorithm (http://r-forge.r-project.org/projects/rniftilib/) calculated the stimulation "entry coordinates" with the shortest distance from the target coordinate in the brain to the head surface for each subject. The coils were placed over these entry coordinates on the surface of the head. The coils targeting PMd or SMG were placed tangentially on the head with the handle pointing at $45^{\circ}$ to the sagittal plane, with the second phase of the biphasic pulse inducing a posterior to anterior current flow in the stimulated cortex.

Data analysis. For each experimental condition, mean RTs and ERs were calculated. For RTs and ERs, separate repeated-measures ANOVAs and post hoc $\mathrm{t}$ tests were performed using SPSS Software (version 13). RTs for trials with correct responses and ERs were separately analyzed for validly and invalidly precued trials and the validity effect (i.e., the difference between invalidly and validly precued trials) using $2 \times 2$ repeatedmeasures ANOVAs including the within-subject factors off-line rTMS (off-line $1 \mathrm{~Hz}$ rTMS of PMd or vertex) and on-line rTMS (effective or sham $10 \mathrm{~Hz}$ rTMS of SMG). A threshold of $\alpha=0.05$ was considered significant for all comparisons. All reported $p$ values are two tailed.

Delta plots for accuracy and RTs. To further explore the differential effects of rTMS over SMG and PMd on accuracy and RTs on invalidly precued trials, we used delta plots within the theoretical framework of a dual-process model and the activation suppression hypothesis (Ridderinkhof, 2002a) to determine effect size as a function of response speed. Dual-process models have been previously used to capture stimulus-response compatibility effects in conflict tasks with delta plots (Ridderinkhof, 2002b; Forstmann et al., 2008a). A schematic representa- 


\section{Experimental design}

\section{A Timeline of the experiment}

session 1

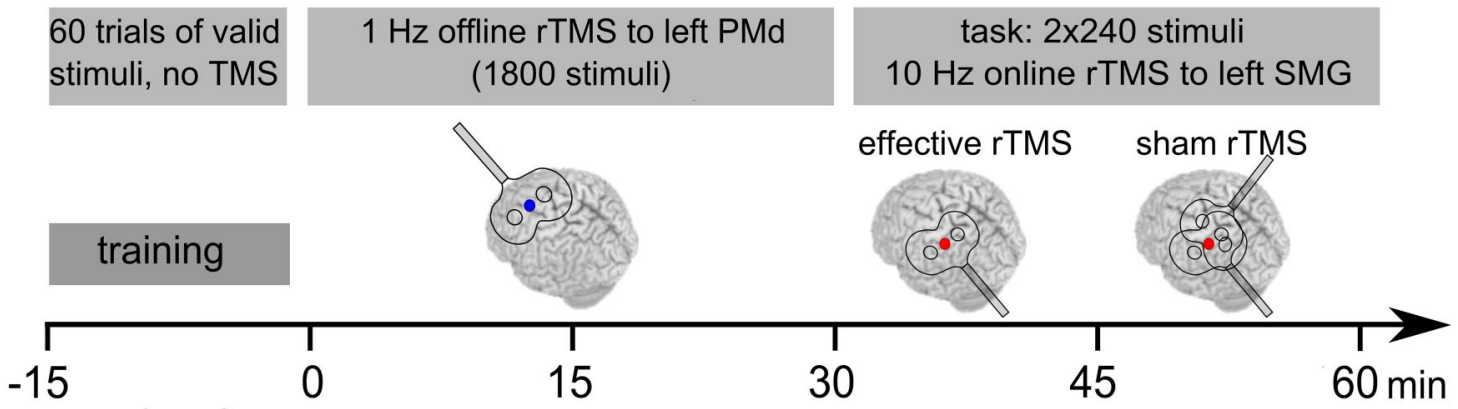

session 2

60 trials of valid stimuli, no TMS
$1 \mathrm{~Hz}$ offline rTMS to vertex (1800 stimuli) task: $2 \times 240$ stimuli

$10 \mathrm{~Hz}$ online rTMS to left SMG

\section{sham rTMS effective rTMS}

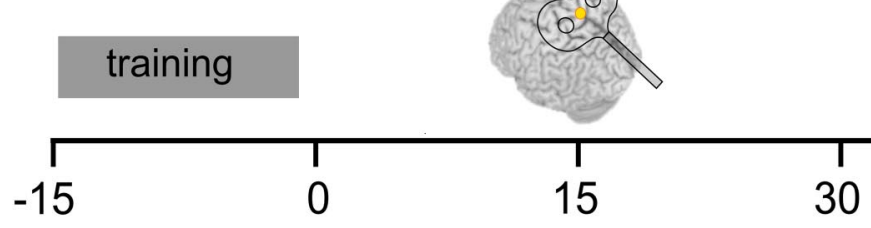

B Experimental task

C Stimulation sites

CR (37.5\%) CL(37.5\%) IR (12.5\%) IL (12.5\%)

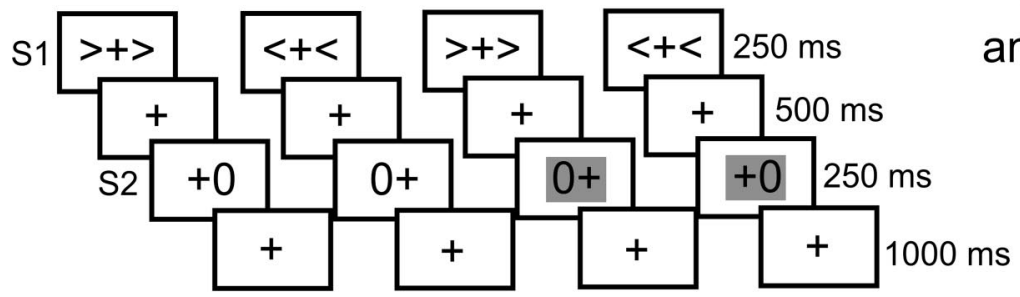

offline rTMS to left PMd

$(\mathrm{x}, \mathrm{y}, \mathrm{z}=-28,8,64 \mathrm{~mm} ; \mathrm{MNI})$

ant

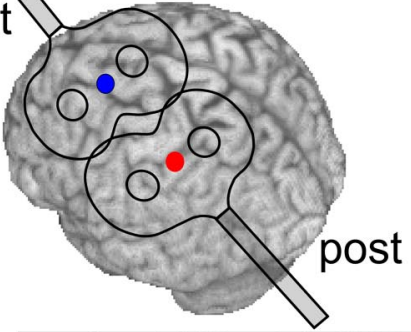

online rTMS to left SMG

$\checkmark$

$\checkmark$

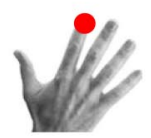

10

$(x, y, z=-52,-36,56 \mathrm{~mm} ; \mathrm{MNI})$

D Example of a single trial

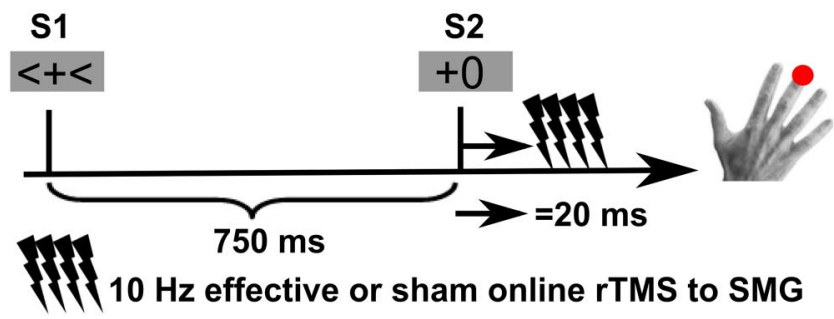

Figure 1. Experimental design. $\boldsymbol{A}$, The experiment consisted of two sessions that were performed at least $5 \mathrm{~d}$ apart in counterbalanced order. In both sessions, $1 \mathrm{~Hz}$ off-line rTMS was applied either to left PMd (blue) or vertex (yellow). During the experimental task, on-line $10 \mathrm{~Hz}$ rTMS was applied to left SMG (red). $\boldsymbol{B}$, Subjects performed a visually precued two-choice RT task. A directional precue (S1) appeared for $250 \mathrm{~ms}$ on the screen and indicated the probable position of a target stimulus (S2). Then $750 \mathrm{~ms}$ after S1, S2 appeared for $250 \mathrm{~ms}$. In $75 \%$ of the trials, the precue validly predicted the target's position and subjects were instructed to prepare for the response indicated by S1. Participants made a spatially compatible button press with their right index finger (left-sided target) or middle finger (right-sided target) according to the position of S2. The order of event types was pseudorandomized. C, Stimulation sites for left PMd and SMG were based on mean stereotactic coordinates obtained from two recent studies (Rushworth et al., 2001; Ward et al., 2010): the PMd site was located at $x, y, z=-28,8$, and $64 \mathrm{~mm}$ and the SMG site at $x, y, z=-52,-36$, and 56 mm within MNI space. $D$, A four-pulse train of $10 \mathrm{~Hz} \mathrm{rTMS} \mathrm{to} \mathrm{left} \mathrm{SMG,} \mathrm{starting} 20 \mathrm{~ms}$ after the onset of $\mathrm{S2}$. ant, anterior; post, posterior. 
Effect of dual-site rTMS over PMd and SMG

Reaction Times

A Valid Precues

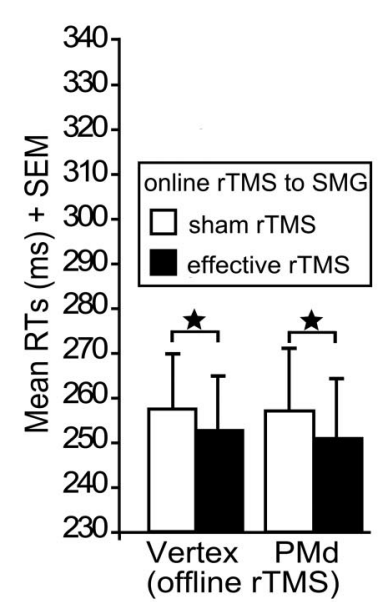

Error Rates (offline rTMS)
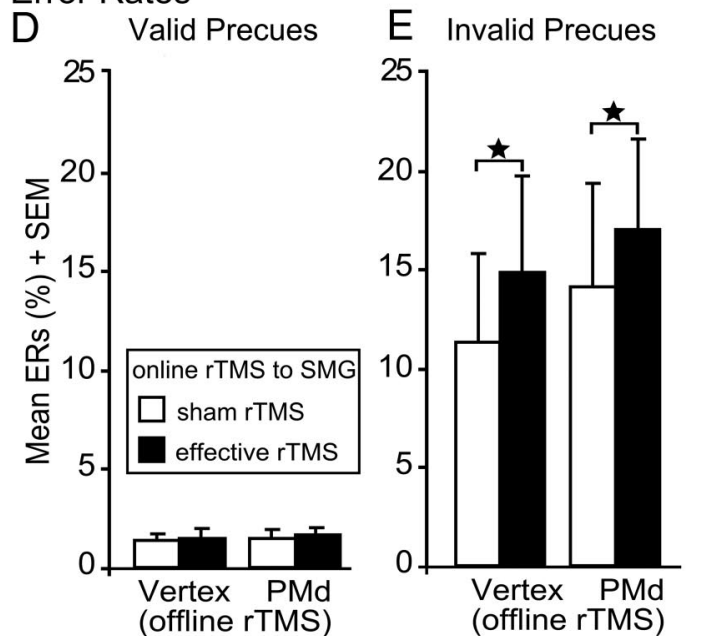

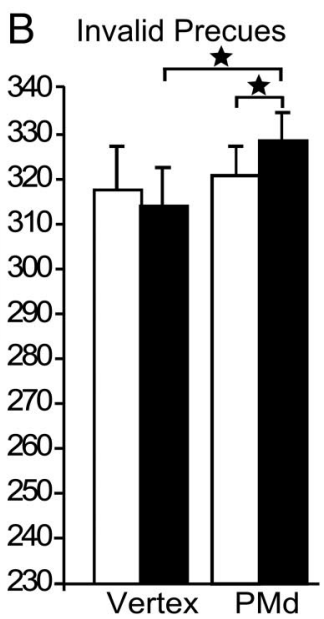

(offline rTMS)

(offline rTMS)
C Invalid-Valid Precues

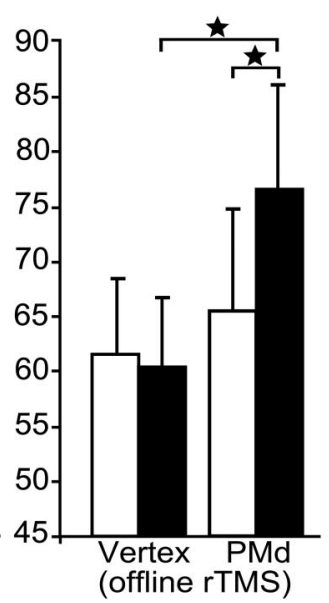

F Invalid-Valid Precues

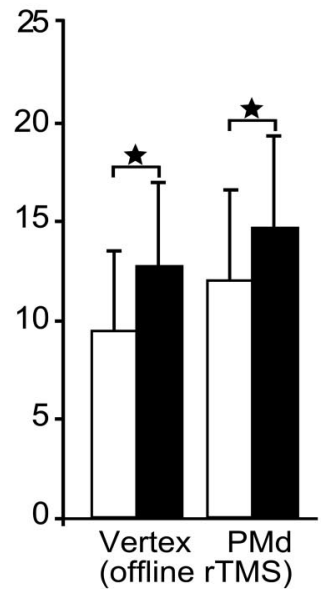

we constructed delta plots for accuracy and RTs by plotting the validity effect (i.e., accuracy or mean RTs on invalidly precued trials versus validly precued trials) as a function of RT (i.e., the average of mean RTs in invalidly and validly precued trials per quartile). Slopes were computed for the delta plot segments connecting the data points of quartile 1 and 2, quartile 2 and 3 , and quartile 3 and 4 (Ridderinkhof, 2002b). Repeated-measures ANOVAs were used to investigate the effects of rTMS on the slopes of the delta plots for accuracy and RTs, including the factors off-line rTMS (1 Hz rTMS of PMd or vertex), on-line rTMS $(10 \mathrm{~Hz}$ effective or sham rTMS over SMG), and slope segment (quartile 1-2, quartile 2-3, and quartile 3-4).

Control experiments. In two follow-up experiments, we tested for the local specificity of the effects evoked by dual-site rTMS targeting the PMv. More specifically, the first control experiment tested whether the priming effect of off-line rTMS over PMd was mediated by PMv, an area previously associated with the processing of visual signals for action planning (Hoshi and Tanji, 2004, 2006; Pardo-Vazquez et al., 2008). If this were the case, dual-site rTMS combining off-line rTMS of PMv with on-line rTMS of SMG should induce a similar or even stronger effect than dualsite rTMS combining off-line rTMS of PMd with on-line rTMS of SMG. The second control experiment addressed regional specificity of the online rTMS effect over left SMG. If the role to efficiently suppress the prepared but invalid response was specific for SMG, we would expect an increase in ERs after on-line rTMS of SMG but not PMv.

\section{Results}

Effects of dual-site rTMS over left PMd and SMG on motor updating

We analyzed the disruptive effects of dualsite rTMS on task processing separately for correct and incorrect responses (i.e., error trials) to investigate whether off-line rTMS of PMd and on-line rTMS of SMG differentially affected RTs and ERs. In val-

tion of our dual-process model is shown in Figure 5A. This model assumes that the controlled processes of stimulus-response translation via a deliberate response activation route are paralleled by a direct activation route. Both routes converge at the level of response activation processes. Within the framework of this model, we assumed that on invalidly precued trials, the direct response activation route would be activated by the invalid precue (S1) whereas the deliberate response decision processes would be activated by the target stimulus (S2).

The activation suppression hypothesis (Ridderinkhof, 2002a) states that the selective suppression of direct stimulus activation takes some time to build up and varies from trial to trial. Given these dynamics, interference effects (i.e., activation of the direct response based on the invalid precue) are more effectively controlled by selective suppression when subjects make slow as opposed to faster responses (Ridderinkhof et al., 2005). The processing dynamics within the dual-route model can be explored by examining the points in time where delta plots diverge between different experimental conditions. Delta plots for accuracy usually have a positive slope indicating accuracy decreases as a function of response speed (i.e., speed-accuracy trade-off).

To construct delta plots for accuracy and response times, RTs for both correct and incorrect responses were rank ordered per rTMS condition for each subject and then divided into four equal-sized RT quartiles. Mean RT and accuracy were determined for each quartile in each condition. Finally, idly precued trials, effective relative to sham on-line rTMS significantly decreased RTs independent of the off-line rTMS site (main effect of on-line rTMS; $F_{(1,19)}=19.77 ; p=0.0001$; Fig. $2 A$ ). On invalidly precued trials, we did not find any main effects on RTs. However, a significant interaction between off-line and on-line rTMS $\left(F_{(1,19)}=8.14 ; p=0.01\right)$ showed that off-line rTMS of left PMd sensitized the ipsilateral SMG to the acute disruptive effects of high-frequency on-line rTMS on response initiation. This finding indicates that a perturbation of both areas was necessary to delay task performance. RTs were significantly increased with effective on-line rTMS to SMG (post hoc paired $t$ test: $t_{(19)}=2.10 ; p=0.045$ ), but not with sham rTMS to SMG ( $p=0.59$ ) after off-line $1 \mathrm{~Hz}$ rTMS of PMd relative to vertex. Of note, the delaying effect of on-line rTMS over left SMG (vs sham) on response initiation critically depended on the type of off-line rTMS, because it only led to RT increases when preceded by off-line rTMS to PMd $\left(t_{(19)}=2.24 ; p=0.04\right.$; Fig. $\left.2 B\right)$ but not off-line rTMS over the vertex.

Combined disruption of both PMd and SMG increased the RT cost for invalidly precued trials (i.e., the validity effect or difference in RTs between invalidly and validly precued trials: 
main effect of off-line rTMS: $F_{(1,19)}=$ 6.03; $p=0.024)$, but the type of off-line rTMS interacted with the type of on-line $\operatorname{rTMS}\left(F_{(1,19)}=10.03 ; p=0.005\right.$; Fig. $\left.2 C\right)$. The significant interaction between offline and on-line rTMS again showed that an intact function of left PMd could compensate for a disruption of ipsilateral SMG on stimulus-driven initiation of the unprepared response unless it was disrupted with off-line rTMS. Consequently, the RT difference between invalidly and validly precued trials was enhanced only if effective on-line rTMS to SMG was preceded by off-line rTMS of left PMd relative to vertex $\left(t_{(19)}=4.44 ; p=0.0001\right)$. This was not the case for sham on-line rTMS to left SMG ( $p=0.35)$. The sensitizing effect of off-line $1 \mathrm{~Hz}$ rTMS over left PMd on the response delay induced with on-line rTMS of left SMG was further substantiated by the finding that effective relative to sham on-line rTMS of left SMG increased RTs only after $1 \mathrm{~Hz}$ rTMS of $\operatorname{PMd}\left(t_{(19)}=3.29 ; p=0.004\right)$ but not vertex rTMS $(p=0.78)$.

Analyses of ERs only included errors of commission as errors of omission were negligible ( $<5 \%$ of all error trials). Here, we did not find any effects of rTMS on validly precued trials (Fig. 2D). However, effective on-line rTMS of SMG significantly interfered with the rapid on-line updating of motor plans when the prepared response based on an invalid precue had to be inhibited and replaced by the appropriate response triggered by the target stimulus (main effect of on-line rTMS: $F_{(1,19)}=14.04 ; p=0.001$; Fig. $\left.2 E\right)$. In contrast to stimulus-driven initiation of the unprepared response, the detrimental effect of effective on-line rTMS on the ability to suppress the prepared but inappropriate response was not influenced by the type of off-line rTMS (i.e., no significant interaction between on-line and off-line rTMS and no main effect of off-line rTMS). The validity effect also showed that effective on-line rTMS impaired the inhibition of the prepared invalid response regardless of the type of off-line rTMS, resulting in significantly increased ERs after effective relative to sham rTMS (main effect of on-line rTMS: $F_{(1,19)}=17.50 ; p=0.001$; Fig. 2F).

\section{Dual-site rTMS does not influence the recency effect for correct responses}

Additional ANOVAs investigated potential influences of the effector (i.e., the responding finger: right middle vs index finger) or the validity of the precue in the preceding trial (i.e., recency effect) on task performance. RT is usually shorter when a member of the same compatibility class is presented on the next trial, i.e., for a valid-valid or an invalid-invalid sequence (Praamstra et al., 1999). Specifically, we wanted to test whether the effect of a previous trial on the following one would be modulated by rTMS. Using mean RTs and ERs as dependent variables, repeated-measures ANOVA did not reveal any significant influence of the responding finger on RTs or ERs (all $p>0.27$ ).

We found a main effect of recency on RTs in validly precued trials only $\left(F_{(1,19)}=13.68 ; p=0.002\right.$; Fig. $\left.3 A\right)$, indicating that RTs for validly precued trials were significantly increased when these trials were preceded by invalidly precued ones regardless of the type of off-line or on-line rTMS. The recency effect did not affect the validity effect or RTs in invalidly precued trials, and there was no interaction with off-line or on-line rTMS in either condition (all $p>0.23$; Fig. $3 B, C$ ).

There was no influence of recency on ERs in validly precued trials (Fig. 3D). However, a main effect of recency in invalidly precued trials indicated that ERs were significantly increased when invalidly precued trials were preceded by validly precued ones $\left(F_{(1,19)}=13.41 ; p=0.002 ;\right.$ Fig. $\left.3 E\right)$. The critical contribution of the left SMG to the rapid on-line updating of motor plans was further corroborated by an interaction between the effect of recency and on-line $\operatorname{rTMS}\left(F_{(1,19)}=5.86 ; p=0.03\right.$; Fig. $\left.3 E\right)$. This interaction indicated that effective compared with sham on-line rTMS over left SMG led to significantly more errors when inval- 


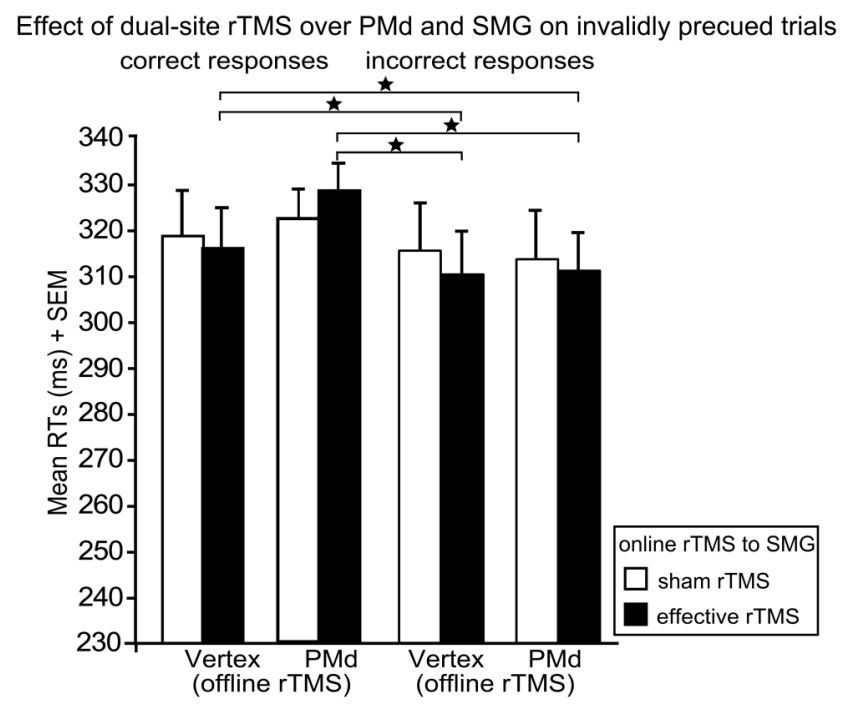

Figure 4. Effect of rTMS over PMd and SMG on correct versus incorrect responses. Mean RTs in invalidly precued trials for correct responses and incorrect responses after off-line rTMS to PMd or vertex and effective or sham on-line rTMS to SMG. The significant two-way interaction between response type and on-line rTMS is shown. Note that the two off-line rTMS conditions are shown separately for illustrating purposes, although the interaction was pooled across the factor off-line rTMS. Error bars indicate onefold SE from the mean (SEM). ${ }^{*} p<0.05$; two tailed.

idly precued trials were preceded by validly precued ones, but this was independent of the type of off-line rTMS $\left(t_{(19)}=2.98 ; p=\right.$ $0.029)$. Comparable results were found for the validity effect: Again, both the main effect of recency $\left(F_{(1,19)}=12.19 ; p=0.003\right)$ and the interaction between on-line rTMS and recency $\left(F_{(1,19)}=\right.$ 5.65; $p=0.03$ ) reached significance, with the latter indicating that effective compared with sham on-line rTMS increased errors when invalidly precued trials were preceded by validly precued ones, but this was independent of the type of off-line rTMS $\left(t_{(19)}=2.87 ; p=0.029\right.$; Fig. $\left.3 F\right)$.

\section{On-line rTMS of SMG decreases RTs for incorrect responses} In an additional analysis, we further investigated the differential effects of off-line rTMS and on-line rTMS on RTs and ERs. To this end, we directly compared RTs for trials with correct versus incorrect responses in invalidly precued trials. The ANOVA yielded a significant interaction between on-line rTMS (i.e., effective vs sham rTMS to SMG) and response type (i.e., correct vs incorrect responses) $\left(F_{(1,19)}=6.86 ; p=0.021\right)$. In invalidly precued trials, effective on-line rTMS of SMG significantly decreased RTs for incorrect responses (i.e., responses based on the invalid $\mathrm{S} 1$ precue) compared with RTs for correct responses (i.e., responses based on the valid S2 target stimulus) $\left(t_{(19)}=3.17 ; p=\right.$ 0.021 ; Fig. 4). Of note, the decrease in RTs for incorrect relative to correct responses was not significant for sham on-line rTMS ( $p=$ 0.18 ). This interaction provided further evidence for a key role of left SMG in suppressing inappropriate response tendencies based on invalid precues. This interaction was independent of the type of off-line rTMS as it did not interact with the factor off-line rTMS, and there were no main effects of on-line or off-line rTMS (all $p>0.12$ ).

Changes in accuracy and RT as a function of response speed The relative increase in ERs for incorrectly precued trials as opposed to correctly precued trials was higher for fast responses. This speed-accuracy relationship is illustrated in the delta plots for accuracy (Fig. 5B). The three-factorial ANOVA, which as- sessed the effects of rTMS on the slopes of the delta plots, showed a main effect of slope segment $\left(F_{(2,18)}=5.73 ; p=0.012\right)$. This was caused by an overall increase in the slopes for the earlier compared with the later RT quartiles across all rTMS conditions (quartile $1-2$ vs quartile $2-3: t_{(19)}=2.45 ; p=0.019$; quartile $1-2$ vs quartile 3-4: $t_{(19)}=3.56 ; p=0.001$ ). While off-line rTMS over PMd did not modify the slopes of the delta plots for accuracy, on-line rTMS over SMG had a consistent effect on the increase in ERs with response speed $\left(F_{(1,9)}=14.04 ; p=0.001\right)$. Relative to sham rTMS, effective on-line rTMS of left SMG caused a stronger increase in ERs with response speed for incorrectly as opposed to correctly precued trials. A significant interaction between the type of on-line rTMS and slope segment showed that effective rTMS of SMG had a differential effect on the speed-accuracy relationship depending on the slope segment $\left(F_{(2,18)}=4.93 ; p=\right.$ 0.02 ). Effective on-line rTMS was associated with steeper accuracy slopes than sham on-line rTMS over SMG for the earlier RT quartiles (quartile 1-2: $t_{(19)}=3.44 ; p=0.003$; quartile 2-3: $\left.t_{(19)}=2.05 ; p=0.055\right)$ but not for the later quartile (quartile 3-4: $p=0.11$ ). This effect of on-line rTMS was not modified by the type of off-line rTMS.

We also constructed delta plots for the RT interference effect (Fig. 5C) and assessed the effects of on-line and off-line rTMS on the change in RT interference effects with a three-factorial ANOVA and the slope of the RT delta plots as dependent variables. This ANOVA showed an interaction between the type of on-line rTMS and slope segment $\left(F_{(2,18)}=4.86 ; p=0.04\right)$. During sham on-line rTMS of SMG, the slopes of the RT delta plots were negative for all slope segments, showing a weaker validity effect of the precue on RT at slower response times (Fig. 5C). This relationship between the RT cost for incorrectly precued trials and response speed was inverted by effective on-line rTMS over SMG. Now the validity effect on RT was more pronounced at slower response times. Accordingly, post hoc $t$ tests showed that effective on-line rTMS of SMG was associated with higher RT slopes than sham on-line rTMS for the early and middle quartiles (quartile 1-2: $t_{(19)}=3.23 ; p=0.004$; quartile $2-3: t_{(19)}=2.33$; $p=0.031)$. A similar trend was also found for the latest quartile (quartile 3-4: $p=0.06$ ).

Relative to off-line rTMS over the vertex, off-line rTMS over left PMd increased the RT cost for invalidly precued trials with increasing response time across all RT segments, but only if the left SMG was additionally perturbed with effective on-line rTMS (Fig. 5C). This interaction between on-line and off-line rTMS was strongest for the middle slope segment (i.e., quartile 2-3). Accordingly, the three-factorial ANOVA revealed a main effect of off-line rTMS $\left(F_{(1,9)}=5.05 ; p=0.035\right)$, a two-way interaction between the type of off-line rTMS and the type of on-line rTMS $\left(F_{(1,9)}=9.07 ; p=0.006\right)$, and a three-way interaction between the type of off-line rTMS, type of on-line rTMS, and the slope segment $\left(F_{(2,18)}=4.95 ; p=0.037\right)$. The sensitizing effect of off-line $1 \mathrm{~Hz}$ rTMS over left PMd on the response delay induced with on-line rTMS of left SMG was further substantiated by post hoc pairwise comparisons. When contrasting the two off-line PMd conditions, effective on-line rTMS of SMG significantly increased the slope for all three slope segments compared with sham on-line rTMS of SMG with the strongest effect on the middle quartile, and this effect was only significant after off-line rTMS of PMd relative to vertex (quartile $1-2: t_{(9)}=4.91 ; p=$ 0.001 ; quartile $2-3: t_{(9)}=6.11 ; p=0.0001$; quartile $3-4: t_{(9)}=$ $4.71 ; p=0.0001)$. 
A Dual-process model for stimulus-response mapping and assumed involvement of the dorsal premotor cortex (PMd) and supramarginal gyrus (SMG)
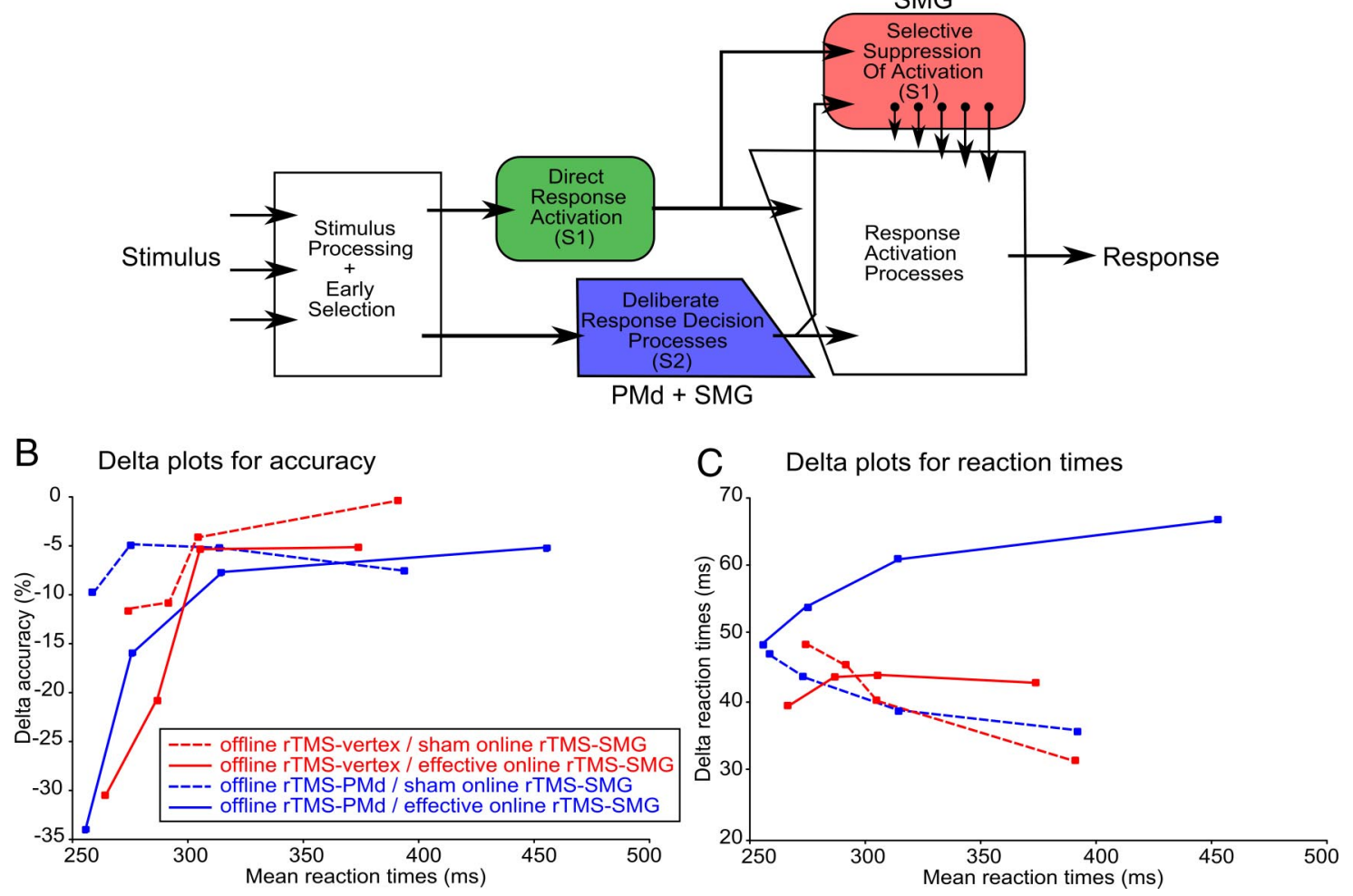

Figure 5. A. Schematic illustration of the dual-process model for stimulus-response mapping in trials with conflicting information. The increasing size of the arrows for selective inhibition represent the operation dynamics for this process (i.e., suppression is not initiated immediately after signal onset but takes time to build up). S1, invalid precue; S2, target stimulus. B, C, Delta plots for the four combinations of off-line and on-line rTMS. The delta plots illustrate the interference effect of invalidly precued trials as a function of response speed. $\boldsymbol{B}$, Relative decrease in accuracy (\%). C, Relative increase in RTs (milliseconds) for invalidly compared with validly precued trials. The four data points of each curve represent the mean data for equally sized RT quartiles ( $0-25 \%$, $26-50 \%, 51-75 \%$, and $76-100 \%)$.

\section{Control experiment 1: off-line rTMS over PMv and on-line rTMS over SMG}

In this experiment $(n=12)$, we asked whether off-line $1 \mathrm{~Hz}$ rTMS of left PMv (vs vertex) would also make additional perturbation of left SMG more likely to delay the successful updating of motor plans. The vertex session was taken from the main experiment. Replicating the results of the main experiment, on-line disruption of left SMG with rTMS significantly decreased RTs in validly precued trials independent of the type of off-line rTMS $\left(F_{(1,11)}=\right.$ $5.75 ; p=0.035$; Fig. 6A). However, neither on-line rTMS of SMG alone nor dual-site rTMS of both PMv (off-line rTMS) and SMG (on-line rTMS) influenced invalidly precued trials. Consequently, dual-site rTMS over left PMv and SMG had no effect on stimulus-driven response initiation in invalidly precued trials (Fig. 6B) or the validity effect (Fig. 6C). This demonstrates the specific role of PMd but not PMv in the type of action reprogramming required by our experimental task.

ERs were not significantly different in validly precued trials (Fig. $6 D$ ). In invalidly precued trials, a main effect of on-line rTMS again confirmed that effective on-line rTMS of SMG interfered with effective suppression of the prepared but invalid responses. Errors for effective relative to sham on-line rTMS to SMG increased for incorrectly precued trials independent of the type of off-line rTMS (main effect of on-line rTMS: $F_{(1,11)}=8.27 ; p=0.015$; Fig. $6 E$ ). The validity effect also confirmed increased ERs for effective relative to sham on-line rTMS of SMG $\left(F_{(1,11)}=7.56 ; p=0.019\right.$; Fig. $\left.6 F\right)$.

To further validate the local specificity of the TMS-induced lesion effects, we directly compared the effects of off-line rTMS over PMd and PMv in invalidly precued trials. A significant interaction $\left(F_{(1,11)}=3.76 ; p=0.044\right)$ between off-line rTMS (rTMS over PMd vs PMv) and on-line rTMS (effective vs sham rTMS of SMG) showed that the increase of RTs with effective on-line rTMS relative to sham on-line rTMS of SMG was found after off-line rTMS of left PMd $\left(t_{(11)}=3.14 ; p=0.043\right)$ but not after off-line rTMS of left PMv $(p=0.21)$.

\section{Control experiment 2: effect of on-line rTMS over left PMv}

An additional experiment tested for the specificity of the on-line lesion effect $(n=6)$. In this experiment, effective or sham on-line rTMS was applied to left PMv during the task without any preceding off-line rTMS. We did not find any disruptive effects of PMv rTMS. On the contrary, relative to sham rTMS, effective on-line rTMS of left PMv significantly decreased RTs in both validly precued trials $\left(t_{(5)}=4.62 ; p=0.007\right.$; Fig. $\left.7 A\right)$ and invalidly precued trials $\left(t_{(5)}=2.82 ; p=0.037\right.$; Fig. $\left.7 B\right)$ but did not affect the validity effect $(p=0.20$; Fig. $7 C$ ) or ERs (all $p>0.12$; Fig. 7D-F).

\section{Discussion}

This study used a dual-site TMS approach, which targeted left PMd (off-line rTMS) and left SMG (on-line rTMS) to examine the causal involvement of these brain areas in rapid action reprogramming. Regardless of the type of preceding off-line rTMS, on-line rTMS of SMG induced a selective increase in errors when subjects had to reprogram their action because of an invalid precue. This indicates a critical involvement of left SMG in effectively 


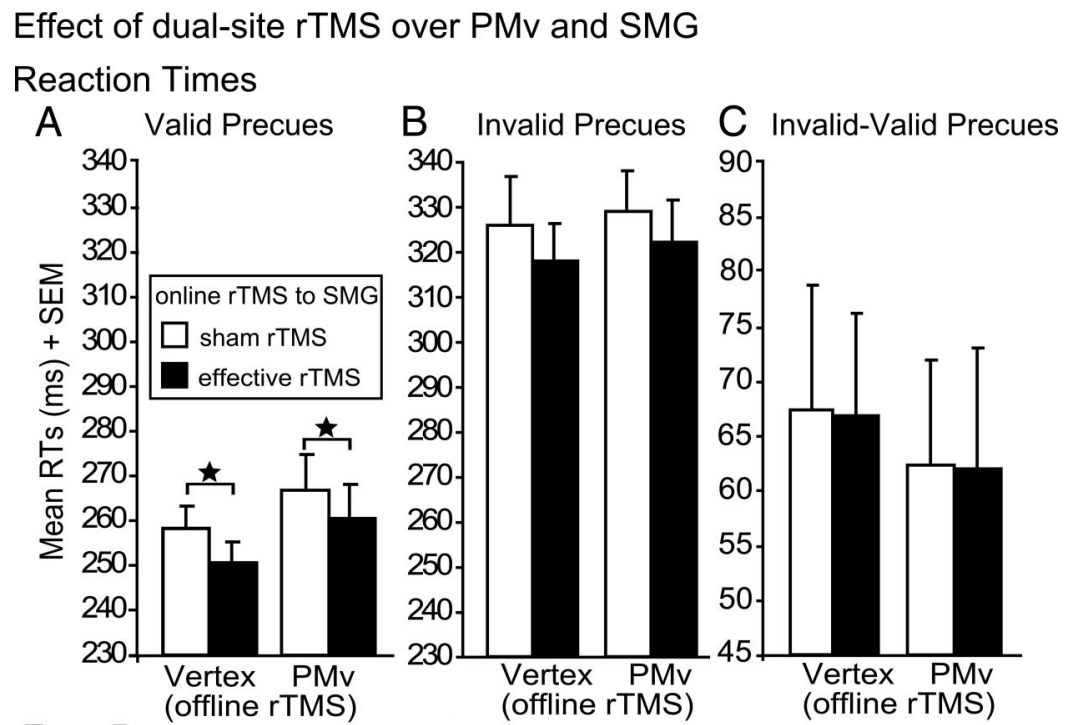

\section{Error Rates}
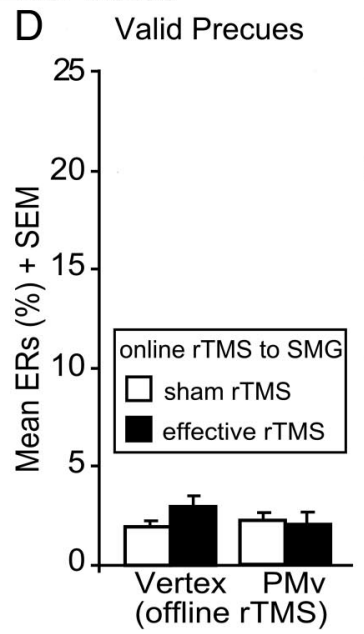

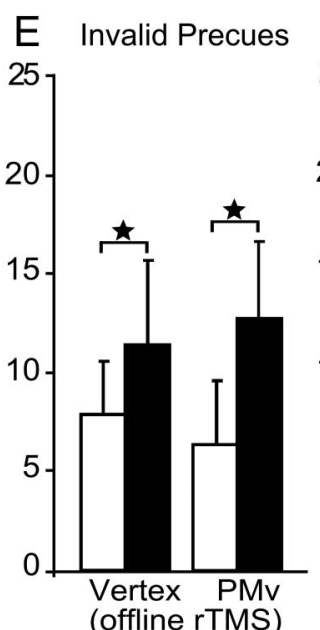

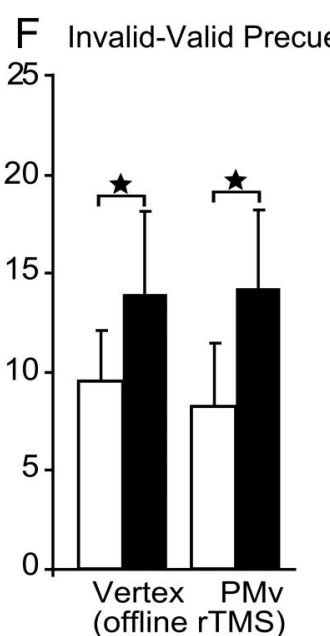

Figure 6. Effect of rTMS over PMv and SMG. Mean RTs and ERs for the different trial types: validly precued trials $(\boldsymbol{A}, \boldsymbol{D})$, invalidly precued trials $(\boldsymbol{B}, \boldsymbol{E})$, and validity effect $(\boldsymbol{C}, \boldsymbol{F})$, after off-line rTMS to PMv or vertex and effective or sham on-line rTMS to SMG. Error bars indicate onefold SE from the mean (SEM). ${ }^{*} p<0.05$; two tailed.

suppressing the release of a prepared but incorrect response. The notion that SMG plays a key role in preventing the release of inappropriate responses extends previous work showing that SMG is important for the on-line control of actions, including the rapid updating of motor plans (Rice et al., 2006; Tunik et al., 2007).

Another key finding of our study is that only a combined disruption of PMd and SMG significantly delayed successful action reprogramming. On-line rTMS over SMG increased RTs for correct but invalidly precued responses, but only after $1 \mathrm{~Hz}$ rTMS had induced an additional dysfunction of PMd. The additional premotor dysfunction increased the functional relevance of SMG during action reprogramming and hereby made the subsequent on-line perturbation of SMG more likely to delay successful action reprogramming. These findings provide causal evidence that left PMd and SMG contribute to the successful updating of motor plans, and that the functional significance of one area for action reprogramming depends on the functional integrity of the other.

The rTMS-induced lesion effects on action reprogramming were anatomically and functionally specific since rTMS failed to show any disruptive effects when given over vertex or PMv. This is somehow surprising as intracortical recordings of neural activity in monkeys have shown that both $\mathrm{PMv}$ and PMd are involved in preparing and selecting actions (Boussaoud and Wise, 1993a,b; Hoshi and Tanji, 2006). However, recent work has demonstrated that cue-related activity of PMv neurons is more closely linked to information about the target location, whereas neurons in PMd encode a combination of information about target location and motor effector (Hoshi and Tanji, 2006). It was hypothesized that $\mathrm{PMv}$ is primarily involved in matching the motor act directly with cue information about target location or target features (direct sensorimotor matching), whereas PMd supports more arbitrary cue-action associations (indirect sensorimotor matching). In accordance with the putative role of PMv in direct sensorimotor matching, a recent dual-site TMS study showed functional interactions between human right $\mathrm{PMv}$ and left primary motor cortex during the reprogramming of naturalistic grasping actions prompted by a change in target size (Buch et al., 2010). In contrast, we did not find evidence for an involvement of $\mathrm{PMv}$ in another form of action reprogramming, namely of switching the motor effector based on invalid "arrow" cues. A possible explanation for the discrepancy is that action reprogramming in our motor paradigm preferentially involved indirect sensorimotor matching subserved by PMd.

On-line and off-line rTMS only affected trials that required action reprogramming. These findings suggest that both SMG and PMd are key regions for the integration of conflicting information. This is compatible with previous studies showing that PMd is involved in nonroutine stimulusresponse mapping (Iacoboni et al., 1998; Dassonville et al., 2001). In contrast, we did not find any evidence for a contribution of SMG and PMd to routine stimulus-response mapping of a prepared response since rTMS did not influence validly precued trials. We attribute this negative finding to the fact that validly precued trials did not require the integration of conflicting information because precue and target triggered the initiation of the same action. Alternatively, the absence of any disruptive effects on validly precued trials might be related to task difficulty. The rTMS effects might have been too weak to efficiently disrupt simple stimulus-response mapping.

Our on-line rTMS train was given shortly after the appearance of the target to interfere with the capability of SMG to inhibit the prepared incorrect response and activate the alternative response based on the target. In the absence of an off-line premotor rTMS session, supramarginal on-line rTMS primarily increased errors in invalidly precued trials. We infer that on-line rTMS disrupted the capability of SMG to efficiently suppress the release of the prepared but inappropriate response. Accordingly, supramarginal on-line rTMS decreased RTs for incorrect responses compared with RTs for correct responses. The interaction between recency effect and on-line rTMS further corroborates the notion 
of a critical contribution of SMG to action reprogramming. The recency effect for invalidly precued trials indicated a strong priming effect of the preceding validly precued trial. It was more difficult to discard the prepared response and replace it with the alternative triggered by the target stimulus when the preceding trial had been validly precued. On-line perturbation of SMG further impaired the correct updating of the prepared motor plan, increasing the recency effect for errors in invalidly precued trials.

Within the framework of a dualprocess model, we assumed that SMG has a key role in the suppression of the precued but incorrect response. Consequently, we found that the increase in errors induced by on-line rTMS over SMG was present when subjects made fast responses. This was evidenced by the delta plots, which relate the validity effect on accuracy to response speed. On-line perturbation of SMG resulted in a steeper increase of the "accuracy slopes" at earlier quantile segments of the RT distribution, suggesting that on-line rTMS of left SMG attenuated the ability to suppress the prepared but incorrect response.

This was further substantiated by our finding that during sham rTMS of SMG, the RT validity effect was stronger for faster response times, whereas the validity effect on RT became more pronounced for slower response times during effective rTMS of SMG. The change in the slopes of the RT delta plots suggests that effective on-line rTMS selectively decreased the suppression of direct response activation, which should have its strongest impact on slow responses (Ridderinkhof, 2002b).

On-line rTMS of SMG also revealed a contribution of left SMG in the initiation of the correct action in response to the target in invalidly precued trials, but only when left PMd had been additionally perturbed by off-line rTMS. The delta plots relating the RT validity effect with response speed confirm that a combined on-line-off-line perturbation of SMG and PMd was necessary to interfere with deliberate response decision processes.

We suggest that the "lesion effect" caused by acute disruption of neural processing in SMG was not sufficient to impair response updating because intact neural processing in PMd was still sufficient to maintain a normal level of deliberate response activation. The additional impairment of local processing in PMd prevented effective compensation and slowed down the initiation of correct responses in invalidly precued trials. Hence, the observed increase in RTs after dual-site rTMS of PMd and SMG can be explained by a summation effect of the "lesion effects" induced by both rTMS protocols. In the context of a pre-existing PMd lesion (induced by off-line rTMS), an additional on-line perturbation increased the total lesion load in the neural network subserving action reprogramming and rendered it more challenging to maintain task performance. Thus, a combined impairment of PMd and SMG was required to result in less efficient deliberate response activation.
Of note, the additional perturbation of PMd did not further enhance errors. This might indicate a preferential cooperation between PMd and SMG in terms of correct stimulus-response mapping, but not for the suppression of inappropriate preplanned responses, which might be supported by other areas.

Previous dual-site TMS studies investigated interhemispheric interactions between homologous areas (Strens et al., 2003; O'Shea et al., 2007), indicating that on-line rTMS over a homolog cortical site can unmask an off-line lesion effect by blocking compensatory processes (Sack et al., 2005). Focusing on intrahemispheric frontoparietal interactions, we show that multiple areas contribute to rapid action reprogramming and that the weight of the relative contribution of a single area can rapidly change, indicating a general flexibility of brain networks in terms of distributed processing.

Our study was motivated by a recent study reporting reduced errors in invalidly precued trials after premotor off-line rTMS. It was suggested that this error reduction was mediated by increased functional coupling between left PMd and SMG (Ward et al., 2010). However, rather than producing a reduction in errors, premotor off-line rTMS increased RTs for correct responses in invalidly precued trials when preceding supramarginal on-line rTMS in our study. Several differences between the two studies may account for the different results. First, we disrupted SMG during task perfor- 
mance, while the previous study did not interfere with task processing in SMG. Second, while subjects were asked to respond as quickly as possible in the previous study, we also put strong emphasis on the accuracy of the response. It is possible that the stronger focus on correct responses might have attenuated possible beneficial effects of premotor off-line rTMS on errors.

In sum, our results provide novel evidence for an intrahemispheric synergistic interaction between left PMd and SMG during action reprogramming. We show that SMG is primarily engaged in the suppression of the prepared but incorrect response. The additional increase in RTs after dual-site rTMS of both PMd and SMG indicates that SMG also supports the initiation of the appropriate action during action programming. However, our results show that the correct response initiation is possible with either an intact PMd or SMG. Only if normal functioning of PMd is impaired by rTMS, does SMG become essential for the rapid initiation of appropriate actions. This demonstrates for the first time that the ability of the motor system to acutely compensate for a transient virtual lesion can be effectively blocked by off-line rTMS to another critical node within the same intrahemispheric network. Hence, the dual-site TMS approach combining off-line and on-line rTMS provides a powerful tool to assess the capacity of the motor system to acutely compensate for focal dysfunction. Specifically, this approach can unravel the causal role of functional interactions within networks, and ultimately might contribute to the understanding of how such networks reorganize after long-term disruption such as occurring after stroke.

\section{References}

Bestmann S, Thilo KV, Sauner D, Siebner HR, Rothwell JC (2002) Parietal magnetic stimulation delays visuomotor mental rotation at increased processing demands. Neuroimage 17:1512-1520. CrossRef Medline

Boussaoud D, Wise SP (1993a) Primate frontal cortex: neuronal activity following attentional versus intentional cues. Exp Brain Res 95:15-27. CrossRef Medline

Boussaoud D, Wise SP (1993b) Primate frontal cortex: effects of stimulus and movement. Exp Brain Res 95:28-40. Medline

Buch ER, Mars RB, Boorman ED, Rushworth MF (2010) A network centered on ventral premotor cortex exerts both facilitatory and inhibitory control over primary motor cortex during action reprogramming. J Neurosci 30:1395-1401. CrossRef Medline

Chambers CD, Bellgrove MA, Gould IC, English T, Garavan H, McNaught E, Kamke M, Mattingley JB (2007) Dissociable mechanisms of cognitive control in prefrontal and premotor cortex. J Neurophysiol 98:3638-3647. CrossRef Medline

Christensen MS, Lundbye-Jensen J, Geertsen SS, Petersen TH, Paulson OB, Nielsen JB (2007) Premotor cortex modulates somatosensory cortex during voluntary movements without proprioceptive feedback. Nat Neurosci 10:417-419. Medline

Dassonville P, Lewis SM, Zhu XH, Ugurbil K, Kim SG, Ashe J (2001) The effect of stimulus-response compatibility on cortical motor activation. Neuroimage 13:1-14. CrossRef Medline

Forstmann BU, van den Wildenberg WP, Ridderinkhof KR (2008a) Neural mechanisms, temporal dynamics, and individual differences in interference control. J Cogn Neurosci 20:1854-1865. CrossRef Medline

Forstmann BU, Jahfari S, Scholte HS, Wolfensteller U, van den Wildenberg WP, Ridderinkhof KR (2008b) Function and structure of the right inferior frontal cortex predict individual differences in response inhibition: a model-based approach. J Neurosci 28:9790-9796. CrossRef Medline

Friston KJ, Ashburner J, Frith CD, Poline J-B, Heather JD, Frackowiak RSJ (1995) Spatial registration and normalization of images. Hum Brain Mapp 3:165-189. CrossRef

Grefkes C, Nowak DA, Wang LE, Dafotakis M, Eickhoff SB, Fink GR (2010) Modulating cortical connectivity in stroke patients by rTMS assessed with fMRI and dynamic causal modeling. Neuroimage 50:233-242. CrossRef Medline

Hoshi E, Tanji J (2004) Functional specialization in dorsal and ventral premotor areas. Prog Brain Res 143:507-511. CrossRef Medline
Hoshi E, Tanji J (2006) Differential involvement of neurons in the dorsal and ventral premotor cortex during processing of visual signals for action planning. J Neurophysiol 95:3596-3616. CrossRef Medline

Iacoboni M, Woods RP, Mazziotta JC (1998) Bimodal (auditory and visual) left frontoparietal circuitry for sensorimotor integration and sensorimotor learning. Brain 121:2135-2143. CrossRef Medline

Mars RB, Klein MC, Neubert FX, Olivier E, Buch ER, Boorman ED, Rushworth MF (2009) Short-latency influence of medial frontal cortex on primary motor cortex during action selection under conflict. J Neurosci 29:6926-6931. CrossRef Medline

Mayka MA, Corcos DM, Leurgans SE, Vaillancourt DE (2006) Threedimensional locations and boundaries of motor and premotor cortices as defined by functional brain imaging: a meta-analysis. Neuroimage 31: 1453-1474. CrossRef Medline

Neubert FX, Mars RB, Buch ER, Olivier E, Rushworth MF (2010) Cortical and subcortical interactions during action reprogramming and their related white matter pathways. Proc Natl Acad Sci U S A 107:13240-13245. CrossRef Medline

Oldfield RC (1971) The assessment and analysis of handedness: the Edinburgh inventory. Neuropsychologia 9:97-113. CrossRef Medline

O'Shea J, Johansen-Berg H, Trief D, Göbel S, Rushworth MF (2007) Functionally specific reorganization in human premotor cortex. Neuron 54: 479-490. CrossRef Medline

Pardo-Vazquez JL, Leboran V, Acuña C (2008) Neural correlates of decisions and their outcomes in the ventral premotor cortex. J Neurosci 28 : 12396-12408. CrossRef Medline

Praamstra P, Kleine BU, Schnitzler A (1999) Magnetic stimulation of the dorsal premotor cortex modulates the Simon effect. Neuroreport 10: 3671-3674. CrossRef Medline

Rice NJ, Tunik E, Grafton ST (2006) The anterior intraparietal sulcus mediates grasp execution, independent of requirement to update: new insights from transcranial magnetic stimulation. J Neurosci 26:8176-8182. CrossRef Medline

Ridderinkhof KR (2002a) Activation and suppression in conflict tasks: empirical clarification through distributional analyses. In: Common mechanisms in perception and action. Attention and performance (Prinz W, Hommel B, eds), pp 494-519. Oxford, UK: Oxford UP.

Ridderinkhof KR (2002b) Micro- and macro-adjustments of task set: activation and suppression in conflict tasks. Psychol Res 66:312-323. CrossRef Medline

Ridderinkhof KR, Scheres A, Oosterlaan J, Sergeant JA (2005) Delta plots in the study of individual differences: new tools reveal response inhibition deficits in $\mathrm{AD} / \mathrm{HD}$ that are eliminated by methylphenidate treatment. J Abnorm Psychol 114:197-215. CrossRef Medline

Rossi S, Hallett M, Rossini PM, Pascual-Leone A (2009) Safety, ethical considerations, and application guidelines for the use of transcranial magnetic stimulation in clinical practice and research. Clin Neurophysiol 120:2008-2039. CrossRef Medline

Rushworth MF, Ellison A, Walsh V (2001) Complementary localization and lateralization of orienting and motor attention. Nat Neurosci 4:656-661. CrossRef Medline

Sack AT, Camprodon JA, Pascual-Leone A, Goebel R (2005) The dynamics of interhemispheric compensatory processes in mental imagery. Science 308:702-704. CrossRef Medline

Schubotz RI (2007) Prediction of external events with our motor system: towards a new framework. Trends Cogn Sci 11:211-218. CrossRef Medline

Siebner HR, Rothwell J (2003) Transcranial magnetic stimulation: new insights into representational cortical plasticity. Exp Brain Res 148:1-16. CrossRef Medline

Siebner HR, Filipovic SR, Rowe JB, Cordivari C, Gerschlager W, Rothwell JC, Frackowiak RS, Bhatia KP (2003) Patients with focal arm dystonia have increased sensitivity to slow-frequency repetitive TMS of the dorsal premotor cortex. Brain 126:2710-2725. CrossRef Medline

Siebner HR, Hartwigsen G, Kassuba T, Rothwell JC (2009) How does transcranial magnetic stimulation modify neuronal activity in the brain? Implications for studies of cognition. Cortex 45:1035-1042. CrossRef Medline

Strens LH, Fogelson N, Shanahan P, Rothwell JC, Brown P (2003) The ipsilateral human motor cortex can functionally compensate for acute contralateral motor cortex dysfunction. Curr Biol 13:1201-1205. CrossRef Medline 
Tunik E, Rice NJ, Hamilton A, Grafton ST (2007) Beyond grasping: representation of action in human anterior intraparietal sulcus. Neuroimage 36 [Suppl 2]:T77-T86.

Verbruggen F, Aron AR, Stevens MA, Chambers CD (2010) Theta burst stimulation dissociates attention and action updating in human inferior frontal cortex. Proc Natl Acad Sci U S A 107:13966-13971. CrossRef Medline
Ward NS, Bestmann S, Hartwigsen G, Weiss MM, Christensen LO, Frackowiak RS, Rothwell JC, Siebner HR (2010) Low-frequency transcranial magnetic stimulation over left dorsal premotor cortex improves the dynamic control of visuospatially cued actions. J Neurosci 30:9216-9223. Medline 